\title{
Formation and evolution of nanoscale calcium phosphate precursors under biomimetic conditions
}

Ludovica M. Epasto ${ }^{\#, s}$, Tristan Georges ${ }^{+, \$}$, Albina Selimovic $^{\#,}$, Jean-Michel Guignier, + Thierry Azaïs, ${ }^{+,}$, Dennis Kurzbach ${ }^{\# *{ }^{*}}$

\#University Vienna, Faculty of Chemistry, Institute of Biological Chemistry, Währinger Str. 38, 1090, Vienna, Austria. ${ }^{+}$Sorbonne Université, CNRS, Laboratoire de Chimie de la Matière Condensée de Paris (LCMCP), 4, place Jussieu, F-75005 Paris, France

${ }^{\dagger}$ Sorbonne Université, Institut de Minéralogie et Physique des Milieux Condensés (IMPMC), 4, place Jussieu, F-75005 Paris, France

\footnotetext{
\$These authors contributed equally

*Corresponding authors: DK: dennis.kurzbach@univie.ac.at; TA: thierry.azais@sorbonne-universite.fr
}

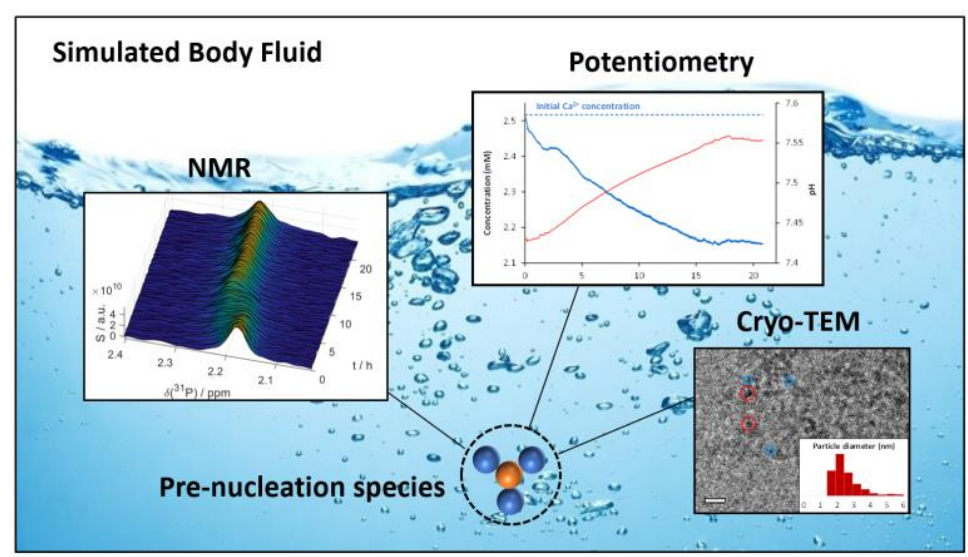




\section{Abstract}

Simulated body fluids that mimic human blood plasma are widespread media for in-vitro studies in an extensive array of research fields, from biomineralization to surface and corrosion sciences. We show that these solutions undergo dynamic nanoscopic conformational rearrangements on the timescale of minutes to hours, even though they are commonly considered stable or metastable. In particular, we find and characterize nanoscale inhomogeneities made of calcium phosphate (CaP) aggregates that emerge from homogeneous SBF within a few hours and evolve into prenucleation species (PNS) that act as precursors in CaP crystallization processes. These ionic clusters consist of $\sim 2 \mathrm{~nm}$ large spherical building units that can aggregate into suprastructures with sizes of over $200 \mathrm{~nm}$. We show that the residence times of phosphate ions in the PNS depend critically on the total PNS surface. These findings are particularly relevant for understanding non-classical crystallization phenomena, in which PNS are assumed to act as building blocks for the final crystal structure. 


\section{Introduction}

Biomineralisation is defined as the ability of living organisms to produce mineral phases embedded mostly in calcified tissues such as bone and teeth of vertebrates. ${ }^{1-3}$ Beyond its fundamental aspect, its understanding is also of imminent interest for developing nature-inspired materials with tailored properties such as improved bone implants ${ }^{4}$ or functional materials. ${ }^{5,6}$ In this context, simulated body fluids (SBF) that mimic the ionic composition of human blood plasma $^{7,8}$ are ubiquitous and used in a wide array of research fields, from the design of bone graft materials $^{9,10}$ and tissue engineering ${ }^{11,12}$ to corrosion and biodegradation studies ${ }^{13-15}$ to bioinspired materials design. ${ }^{16,17}$ In particular, SBF plays its crucial role as it is the most widespread biomimetic medium used to assess the bioactivity of materials. ${ }^{18-20}$ Notwithstanding its frequent use, the "nanostructure" of SBF remains to large degrees unsolved. A deeper understanding of the structural dynamics is yet essential due to the influence of solvent properties on mineralization and crystallization phenomena. ${ }^{21,22}$ Indeed, environmental conditions often determine the morphology of solid phases precipitating from solution.

In this regard, SBF have gained particular attention as a result of the recent observation of so-called prenucleation species (PNS) and their involvement in the early onset of biomimetic calcium phosphate precipitation (e.g. hydroxyapatite). ${ }^{23,24}$ These PNS are highly dynamic nanometric ionic clusters that spontaneously form in solution preceding the precipitation of solid crystalline phases. $^{25}$ Today, PNS have been documented for calcium phosphates $(\mathrm{CaP})^{21,23,26-29}$ and carbonates $(\mathrm{CaC})^{30-33}$ as well as for iron oxides. ${ }^{34}$ Interestingly, their involvement in the formation of biominerals challenges the long-standing nucleation-and-growth paradigm, ${ }^{35,36}$ which has stimulated an ongoing update of the existing crystallization theories. $^{26,37,38}$

In addition, it has been suggested that PNS structure and dynamics determine the crystallization path a material takes. ${ }^{22}$ Hence, a better understanding of PNS behavior in SBF promises new avenues for the rational design of solid biomimetic ceramics via control over the initial reaction conditions that regulate the molecular architecture of the final material. However, the structure and dynamic behavior of PNS (in particular in SBF) remain poorly understood, and the factors that control the associated nucleation events remain unclear, leaving significant gaps in the current understanding of crystallization. 39,40 a)

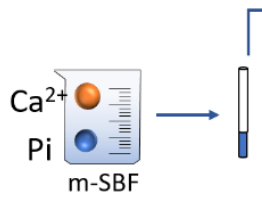

b)

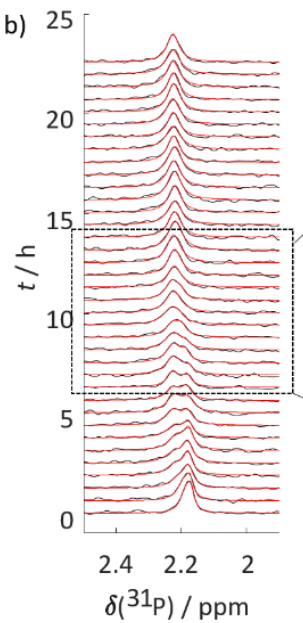

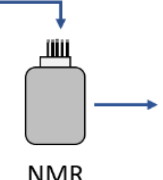

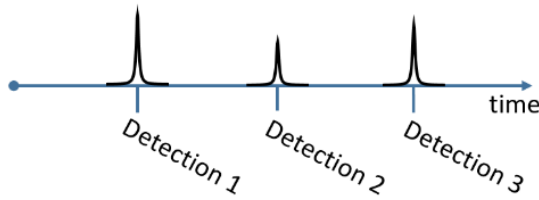

c)

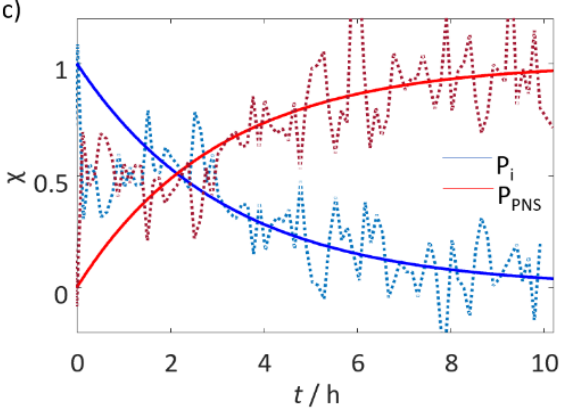

Figure 1: Monitoring of PNS formation in $\mathrm{MSBF}$ through real-time NMR. a) Scheme of experimental workflow. The evolution of PNS at $37^{\circ} \mathrm{C}$ in $\mathrm{mSBF}$ is traced by consecutive detections of ${ }^{31} \mathrm{P}$ spectra. b) ${ }^{31} \mathrm{P}$ NMR signals detected for mSBF over a period of $24 \mathrm{~h}$ (black) and corresponding fits to two Lorentzian functions (red). Initially, only a single resonance can be observed. After $\sim 5 \mathrm{~h}$ a second peak appears. Between 8 and $10 \mathrm{~h}$, the first and second peaks merge forming a single signal. The insert highlights the transition period during which the two signals merge. The resulting single line resonates at a frequency between those of the precursor signals. At $t>15 \mathrm{~h}$ again only a single line is visible. Note that the spectra are normalized. c) Time-dependence of the fractional contribution $\chi$ of the two lines to the overall spectrum. The contribution of free phosphate is shown in blue and that of PNS-bound phosphate in red. After $10 \mathrm{~h}$ the signals merged and the two contributions could not be separated anymore. 
Indeed, the highly dynamic behavior of SBF and its ionic constituents is challenging to characterize experimentally from both, structural and dynamical viewpoints. To overcome this bottleneck, we here present an integrative approach, including the first observation of PNS by real-time nuclear magnetic resonance (NMR) spectroscopy in solution combined with cryoelectron microscopy and calcium potentiometric measurements. We shed light on the constitution of SBF at the nanoscale revealing dynamical and structural inhomogeneities therein and thus providing a significant advance in their understanding.

In particular, we show how SBF undergo defined and irreversible molecular rearrangements with time, challenging the long-standing notion of its (meta)stability. ${ }^{23,41,42}$ We find that nanometric calcium phosphate (CaP) clusters with diameters of ca. $2 \mathrm{~nm}$ form within 5 hours after preparing fresh SBF. We identify these clusters as previously described calcium phosphate-based PNS. $^{23}$ Subsequently, these PNS can aggregate to form soluble supra-structures of several hundreds of nanometers in size that might precede the precipitation of solid CaP. We find calcium triphosphate units to be the basic units of the PNS. Furthermore, we characterize the exchange kinetics of phosphates between PNS-bound and free states, demonstrating that the frequency of exchange depends critically on the quantity of PNS in solution.

\section{Results and Discussion}

In the following, we will first describe the formation and internal dynamics of PNS in SBF employing real-time NMR. Secondly, we describe the PNS constitution by means of transmission electron microscopy (TEM) and potentiometric experiments.

We want to stress that we herein collectively term all CaP aggregates that occur in solution during the early onset of $\mathrm{CaP}$ precipitation, for the sake of generality, prenucleation species or 'PNS'. This notion should here be understood as neutral, i.e., as not referring to any nucleation or crystallization theory. ${ }^{26,37-39}$

Real-time NMR of evolving SBF
We initially observed the formation and evolution of PNS by real-time NMR of modified $\mathrm{SBF}^{43,44}$ ( $\mathrm{mSBF}$ which provides improved $\mathrm{pH}$ stability over neat SBF). Capitalizing on the strong dependence of the chemical shift of phosphate ions on their local environment, these experiments could distinguish the signals of phosphates bound in a PNS from free phosphates in solution. They revealed that PNS form within five hours after preparing fresh $\mathrm{mSBF}$ and undergo continuous changes throughout a $15 \mathrm{~h}$ period.

We recorded a series of ${ }^{31} \mathrm{P}$ NMR spectra as outlined in Fig. 1a, starting immediately after the sample preparation. Fig. $1 \mathrm{~b}$ shows the resulting spectra (black lines) and superimposed fits (red lines) to two Lorentzian functions that model the NMR line shape (see the Experimental section for details). At $t=0$, a single resonance at $2.18 \mathrm{ppm}$ is detectable, corresponding to free inorganic phosphate $\left(\mathrm{P}_{\mathrm{i}}\right)$ dissolved in mSBF similar in position and linewidth to the control experiment (Fig. S1). After 3-5 h, a second broader peak appears at 2.23 ppm. We assign this signal to emerging PNS (vide infra) and denote the involved phosphates henceforth as $\mathrm{P}_{\mathrm{PNS}}$. During this period, our fitting routine can quantitatively reproduce the spectra with only two lines. From $8 \mathrm{~h}$ to $10 \mathrm{~h}$ after preparation, both signals are broadened compared to their initial line width and slowly approach each other.

After $10 \mathrm{~h}$, the signals merge, and only a single line remains at a chemical shift of $2.21 \mathrm{ppm}$ (insert Fig. 1b). Notably, the total signal intensity is constant throughout the detection period (up to 30 h), indicating that calcium phosphate does not precipitate from $\mathrm{mSBF}$ under our conditions ( $\mathrm{T}=$ $37^{\circ} \mathrm{C} ; \mathrm{pH}=7.4$ ) throughout the experiment.

Fig. 1c shows the relative fractions $\chi$ of the two
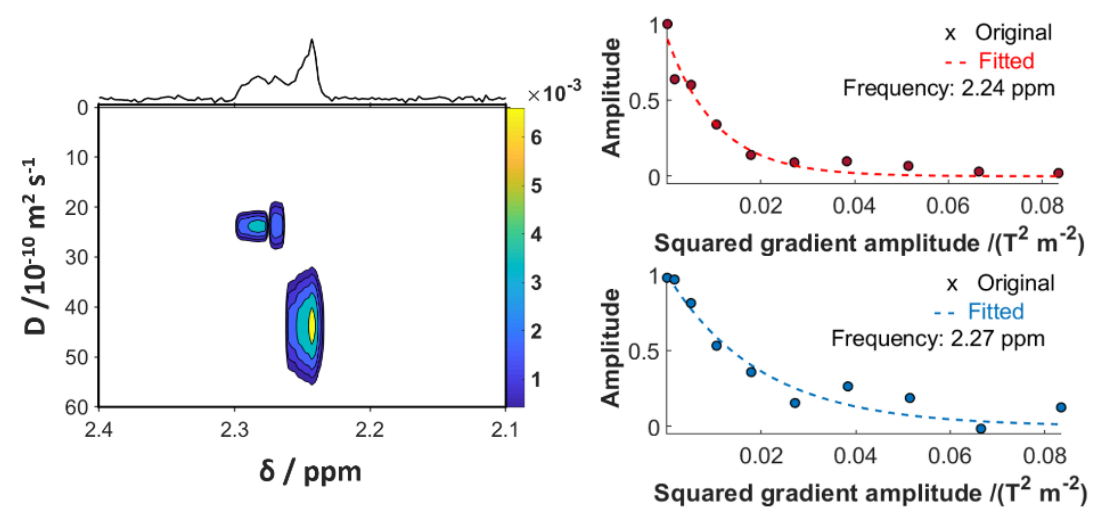

Figure 2: DOSY analysis of mSBF. a) Mapped diffusion coefficients in dependence of the chemical shift. When both, the $P_{1}$ and the $P_{\text {PNS }}$ signals, appear well-separated in the ${ }^{31} \mathrm{P} N M R$ spectra, DOSY of the system allows one to discern two phosphate species via their diffusion coefficients. A slower diffusion coefficient is observed for the species underlying $\mathrm{P}_{\mathrm{PNS}}$ (left) compared the signal underlying $P_{i}$ (right). The diffusion coefficients were $\sim 24 \times 10^{-10} \mathrm{~m}^{2} \mathrm{~s}^{-1} \mathrm{vs}$. $\sim 43 \times 10^{-10} \mathrm{~m}^{2} \mathrm{~s}^{-1}$, respectively. The data were acquired at $37^{\circ} \mathrm{C}$ for $5 \mathrm{~h}$ at a phosphate concentration of $5 \mathrm{mM}$. b) Monoexponential fits of the two DOSY profiles underlying the data in panel (a). The $\mathrm{P}_{\mathrm{PNS}}$ signal decays slower with increasing gradient amplitude than the $\mathrm{P}_{\mathrm{i}}$ 
spectral components of $\mathrm{P}_{\mathrm{i}}$ and $\mathrm{P}_{\mathrm{PNS}}$ of the spectra during the initial period of evolution. The figure highlights how $\mathrm{P}_{\mathrm{i}}$ is consumed to form $\mathrm{P}_{\mathrm{PNS}}$.

\section{Diffusion-ordered spectroscopy provides evidence of PNS}

To characterize the two different phosphate species further, we employed diffusion ordered spectroscopy (DOSY). It showed that the hydrodynamic radius of the PNS is ca. 1.7-fold larger than that of free $\mathrm{P}_{\mathrm{i}}$.

Due to the low signal intensities, these experiments had to be acquired at a $P_{i}$ concentration of $5 \mathrm{mM}$ (a 5 -fold increase compared to neat $\mathrm{mSBF}$ ). However, the results can be safely qualitatively interpreted as, similarly to $\mathrm{mSBF}$, two distinct resonances appear after 10 to $15 \mathrm{~h}$ of maturation (Fig. S2). Fig. 2 displays the DOSY analysis of modified mSBF acquired over a 5 $\mathrm{h}$ period (10h-15h after sample preparation). Notably, two distinct diffusion coefficients are evidenced. A coefficient of $D=\sim 24 \cdot 10^{-10} \mathrm{~m}^{2} \mathrm{~s}^{-1}$ could be observed for the single $P_{\text {PNS }}$ peak. The $P_{i}$ signal yielded to a faster coefficient of $\sim 42 \cdot 10^{-10}$ $\mathrm{m}^{2} \mathrm{~s}^{-1}$. Solving the Stokes-Einstein relation assuming spherical particles, we find that the average hydrodynamic radius $R_{h}$ of $\mathrm{P}_{\mathrm{PNS}}$ is $\sim 1.7$-fold larger than that of $\mathrm{P}_{\mathrm{i}}$, amounting to $\sim 1.0 \mathrm{~nm}$ and $\sim 0.6 \mathrm{~nm}$, respectively. Therefore, the volume of the detected particles is ca. four-fold larger than that of free $P_{\mathrm{i}}$. Hence, we deduce that the second signal appearing in the ${ }^{31} \mathrm{P}$ spectra in Fig. 1 indeed stems from phosphate units embedded in larger ionic clusters that, despite their size, remain in solution, i.e., in a PNS.

\section{Theoretical modeling of the phosphate exchange}

To better understand the $R_{h}$ variations and the real-time NMR data, we theoretically modeled our data assuming two-site chemical exchange. We found that the mSBF system passes from a slow to an intermediate to a fast exchange regime with growing PNS concentration. This circumstance causes the apparent spectral changes in the NMR time series.

To form PNS, it is necessary that the phosphates exchanges between the pool of free $P_{i}$ in solution and the pool of newly bound species $P_{\text {PNS. This }}$ process influences the shape of the NMR spectra in Fig. 3a (similar to $1 b$ ). The time series starts with a single $P_{i}$ signal followed by the emergence of a well-distinguishable $\mathrm{P}_{\mathrm{PNS}}$ signal. Later, both signals first broaden and then merge into a single, again sharp, resonance. This behavior is typical for exchange-driven spectral coalescence. ${ }^{45}$ Fig. $3 c$ illustrates how both signals are broadened and reduced in amplitude during the period of intermediate exchange
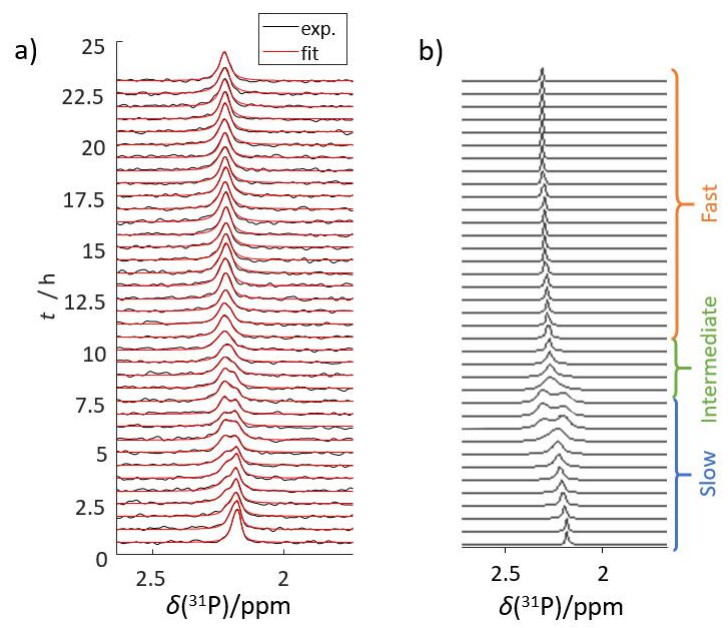

c)

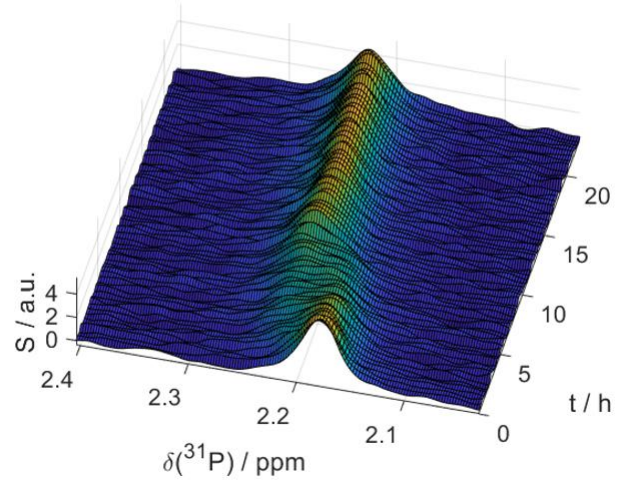

Figure 3: Comparison of a) the experimental results of $\mathrm{mSBF}$ evolution at $37^{\circ} \mathrm{C}$ with b) simulated data. The prediction was performed with a dynamic 2-site exchange model, as described in the main text. In both, the second species appears at ca. 3$5 \mathrm{~h}$, following the exchange path of the NMR detected peak. The brackets indicate the different exchange regimes. c) Nonnormalized surface plot of the ${ }^{31} \mathrm{P} N \mathrm{NMR}$ signal vs. time. The representation illustrates that the signals are broadened and reduced in amplitude during the transition from slow to fast exchange.

We could model this behavior using the Norris equation $^{46}$ for two-site exchange, which can be considered as a simplified version of the CarverRichards equation: ${ }^{47}$

$$
\begin{gathered}
\rho_{T}=\left[\begin{array}{lll}
-i C & \sum_{l} F_{l}
\end{array}\right]\left[1+\left(\frac{1}{\tau}\right) \sum_{l} F_{l}\right]^{-1} \\
F_{l}=n_{l}\left[i\left(\omega-\omega_{k}\right)-\frac{1}{\left(T_{2}\right)_{l}}-\frac{1}{\tau}\right]^{-1}
\end{gathered}
$$

$\rho_{T}$ is the resulting spectrum, $\tau$ stands for the average inverse exchange rate $k_{\mathrm{ex}}$, and $n_{l}$ is the fractional population of the $l^{\text {th }}$ site. $\omega$ is the resonance frequency and $\frac{1}{\left(T_{2}\right)_{l}}$ is the linewidth of the $l^{\text {th }}$ species in the absence of exchange. $C$ is an 
overall proportionality constant assumed here to be 1.

The exchange rate constant $1 / \tau$ was modeled by combining the classical collision theory-Arrhenius law with a first-order kinetic equation:

$$
\begin{aligned}
& Z=n_{P i} n_{P N S} R_{h, P N S} \sqrt{\frac{8 k_{b} T}{\pi m_{r e d}}} \\
& 1 / \tau=Z \exp \left(-\frac{E_{A}}{R T}\right) \\
& \frac{\partial\left[n_{P i}\right]}{\partial t}=-k\left[n_{P i}\right]
\end{aligned}
$$

Where $n_{P i}$ and $n_{P N S}$ indicate the concentrations of free and PNS-bound phosphates, respectively. $R_{h, P N S}$ is the estimated radius of hydration of a PNS (assumed to be $1 \mathrm{~nm}$, in line with the DOSY experiments). $k_{b}$ is the Boltzmann constant, $T$ is the temperature of the system and $m_{\text {red }}$ is the reduced PNS mass. $E_{A}$ is the activation energy and $R$ is the gas constant. In eq. (3) $k$ is the kinetic constant of the conversion $\mathrm{P}_{\mathrm{i}} \rightarrow \mathrm{P}_{\mathrm{PNS}}$.

Combining eqs. 1 to 3 , we could reproduce the experimental data as shown in Fig. $3 a$ and $3 b$. At this stage, the following understanding emerges of the exchange processes in $\mathrm{mSBF}$ :

Initially, aggregation of $\mathrm{P}_{\mathrm{i}}$ and $\mathrm{Ca}^{2+}$ ions leads to CaP-PNS formation and the emergence of a corresponding second signal. At this time, the system is in slow exchange as the number of PNS is still small, compared to free $\mathrm{Pi}$, such that only a small 'exchange surface' is available and a small number of encounters (cf. eq. 2; the pre-factor $n_{P N S}$ is small, leading to slow exchange rates $1 / \tau$ ). After 5 hours, the two peaks broaden as the system undergoes a transition into an intermediate exchange regime where $\Delta \delta\left({ }^{31} P\right)=1 / \tau=k_{e x}$. In other words, the exchange surface grows together with the number of PNS, $n_{P N S}$. Finally, the two peaks merge after ca. $15 \mathrm{~h}$, with a chemical shift averaged between the two initial lines (cf. Fig. 1b). For this case, the model calculations indicate that the phosphates experience a fast exchange between both states, i.e., $\Delta \delta\left({ }^{31} P\right)<1 / \tau$.

The real-time NMR data, diffusion measurements, and theoretical predictions thus suggest a process in which i) PNS form in mSBF spontaneously after its preparation and continue emerging over a time of $>15 \mathrm{~h}, \mathrm{ii}$ ) the diameter of the PNS detected by solution-state NMR is on the order of $2 \mathrm{~nm}$ and iii) that the exchange between freely dissolved and PNS-bound phosphates accelerates when the concentration of PNS grows.

During the slow exchange phase, the chemical shift difference between the two species amounts to $0.05 \mathrm{ppm}$, which corresponds to an exchange rate of $k_{\mathrm{ex}}<10 \mathrm{~Hz}$. Interestingly, earlier studies on

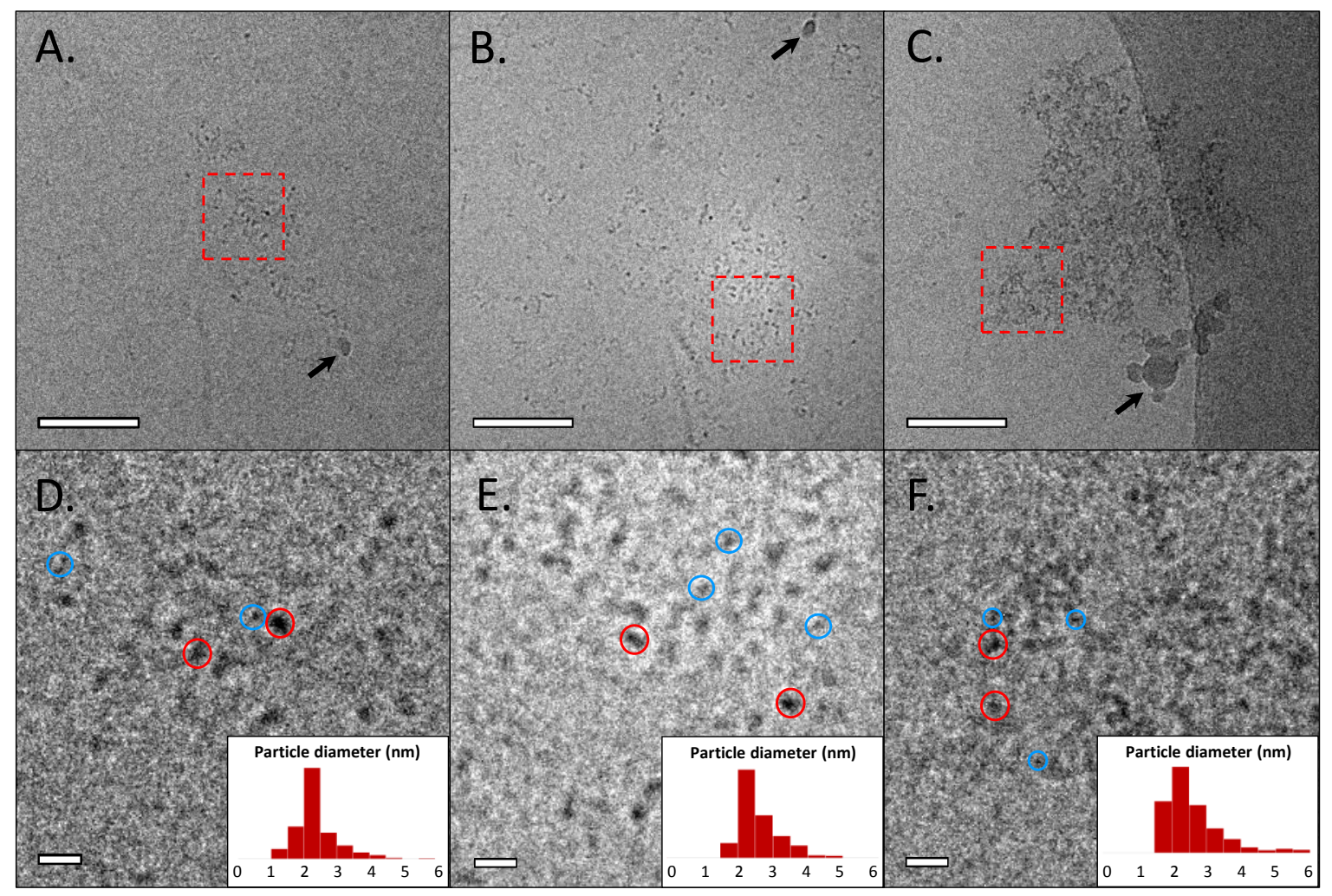

Figure 4: Cryo-TEM observations of mSBF solution at different time points - (A) $1 \mathrm{~h}$, (B) $5.5 \mathrm{~h}$ and (C) $23 \mathrm{~h}$ min after mSBF preparation, respectively. (D-F) Zoom of the corresponding zones delimited by the red squares in (A-C). Insert displays size evaluation of individual PNS. Blue and red circles give example of smaller and bigger PNS, respectively. The black arrows indicate the presence of polluting agents, due to the freezing process. Scale bar $(A-C)=50 \mathrm{~nm}$. Scale bar $(D-F)=10 \mathrm{~nm}$. 
CaP precipitation (dicalcium phosphate dehydrate DCPD) ${ }^{48}$ under non-biomimetic conditions $(\mathrm{pH} 8$ and ion concentrations $>10 \mathrm{mM}$ ) reported much higher limits of $k_{\text {ex }}<250$ to $350 \mathrm{~Hz}$ highlighting the importance of environmental conditions for crystallization studies.

Cryo-TEM analysis reveals PNS supra-structures To further analyze the PNS structure, we performed cryo-TEM observations. Data were acquired $1 \mathrm{~h}, 5.5 \mathrm{~h}$, and $23 \mathrm{~h}$ after sample preparation, following the exchange regimes derived by NMR (Fig. 4 and Fig. S3). After $1 \mathrm{~h}$, we observed the appearance of the first PNS (Fig. 4a). The number of PNS visibly increased after $5.5 \mathrm{~h}$ (Fig. 4b-4c), supporting the time-dependent emergence of PNS in mSBF. After $23 \mathrm{~h}$ of maturation, some PNS are found to aggregate into higher order structures of several hundreds of nanometers including typically $\sim 100$ to 400 PNS. (Fig. 4c). In addition, free PNS were also observed to coexist in solution (Fig. S3).

Higher magnification allows the evaluation of PNS size and distribution (Fig. 4d-f). After $1 \mathrm{~h}$ and $5.5 \mathrm{~h}$, individual PNS with sizes ranging from 1.5 to 3.5 $\mathrm{nm}$ were observed in good agreement with the DOSY NMR data. After $23 \mathrm{~h}$, PNS aggregates were found constituted by individual spherical units again with diameters of 1.5 to $3.5 \mathrm{~nm}$. In addition, bigger units of 4-6 $\mathrm{nm}$ in size were found to coexist at this time of maturation (red circles vs blue circles in Fig. 4d-f).

The observed aggregates and clusters remain in solution in $\mathrm{mSBF}$ for an extended period before CaP precipitation takes place. Hence, these structures likely define the starting point of the pathway $\mathrm{CaP}$ takes towards its solid crystalline state. $^{23,24,27}$

Note that solution-state NMR is limited to aggregates of a few nanometers due to linebroadening effects for bigger molecular assemblies. It is very likely that the detected $\mathrm{P}_{\mathrm{PNS}}$ resonance through ${ }^{31} \mathrm{P}$ NMR species do not reflect all PNS observed by cryo-TEM. Larger species might not be detected. The determined $R_{h}$ of $\sim 1 \mathrm{~nm}$ through DOSY NMR corroborates this assumption.

\section{EDXS analysis reveal phosphate-rich PNS}

We employed energy-dispersive X-ray spectroscopy (EDXS) of mSBF $23 \mathrm{~h}$ after preparation to determine the PNS composition after complete equilibration. In the electronmicrograph, we selected a region of $\sim 1 \mu \mathrm{m}^{2}$ containing a large PNS aggregate (Fig. 5a) and another region void of any PNS (Fig. $5 \mathrm{~b}$ ) as a negative control.
The $\mathrm{Ca}-\mathrm{K} \alpha$ and $\mathrm{Ca}-\mathrm{K} \beta$ peaks are both visible in absence and presence of PNS. In opposition, P-K $\alpha$ peak is only visible in the presence of PNS, indicating the abundance of phosphorous atoms within the ionic clusters. Interestingly, $\mathrm{S}-\mathrm{K} \alpha$ and $\mathrm{Na}-\mathrm{L} \alpha$ signals (markers of sulfate and $\mathrm{Na}^{+}$), of comparable intensity to the $\mathrm{P}-\mathrm{K} \alpha$ peak, are present in both cases (Fig. 5a-b).
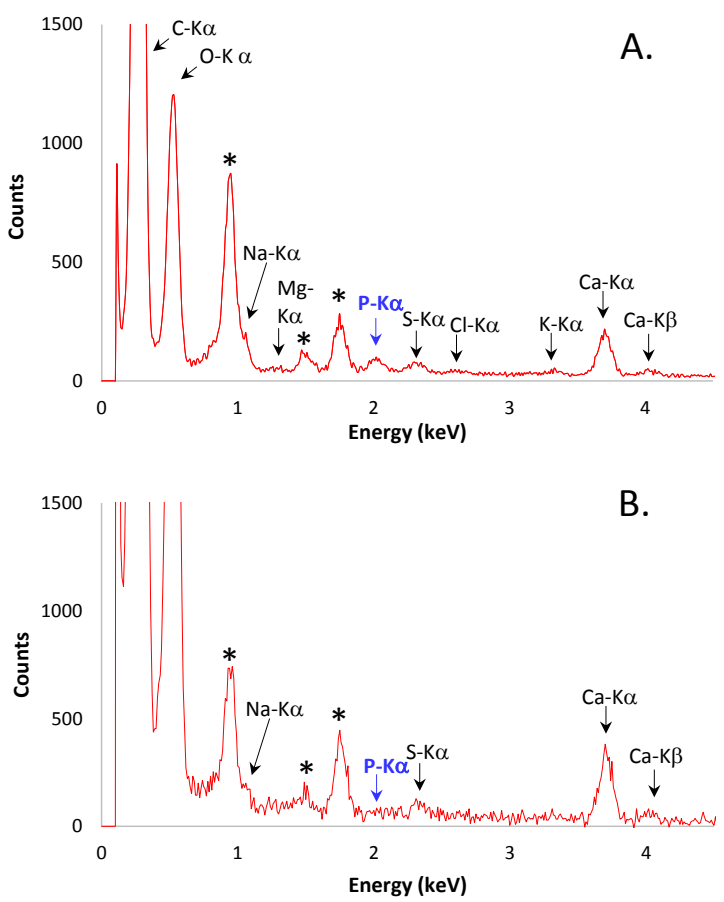

Figure 5: EDXS analysis of mSBF performed $23 \mathrm{~h}$ after preparation. A) The selected zone contains a large amount of PNS. B) The selected zone does not contain a significant amount of PNS (Fig. S4). The electron beam area is $\sim 1 \mu \mathrm{m}^{2}$. Black stars at $0.93,1.49$ and $1.74 \mathrm{keV}$ indicate $\mathrm{Cu}-\mathrm{L} \alpha, \mathrm{Al}-\mathrm{K} \alpha$ and $\mathrm{Si}-\mathrm{K} \alpha$ peaks, respectively, corresponding to the grid, its plasma treatment or pollutions.

Thus, the EDXS results suggest that $\mathrm{mSBF}$, once equilibrated, is depleted in free $P_{i}$ while most phosphate ions are localized within the PNS units. These data are consistent with the ${ }^{31} \mathrm{P}$ NMR data in which the detected fraction of free $\mathrm{Pi}$ is very small at $\mathrm{t}>15 \mathrm{~h}$.

$\mathrm{Ca}^{2+}$ potentiometry reveal PNS Ca/P ratio

To further corroborate the NMR-derived kinetics and determine the protonation state of the phosphate units, we potentiometrically determined the calcium ion concentration over a period of $21 \mathrm{~h}$ following $\mathrm{mSBF}$ preparation.

Fig. 6 displays the time-dependence of the free $\mathrm{Ca}^{2+}$ ion concentration throughout our experiments. After mSBF preparation, the concentration continuously decreases until it reaches a steady-state at $t>16 \mathrm{~h}$. This decrease results from the uptake of $\mathrm{Ca}^{2+}$ ions by the PNS (in 
line with the NMR and TEM data). The proportion of bound calcium ions after ca. $16 \mathrm{~h}$ amounts to $14 \%$ of the initial $\mathrm{Ca}^{2+}$ concentration, i.e., the free ion concentration drops from 2.5 to $2.15 \mathrm{mM}$ (Fig. 6). In return, the amount of bound $\mathrm{Ca}^{2+}$ is $\mathrm{ca} .0 .35$ $\mathrm{mM}$ (Fig. S5). Moreover, from the NMR experiments and EDXS analysis, we can estimate the amount of $\mathrm{P}_{\mathrm{PNS}}$ after $21 \mathrm{~h}$ to $c a .1 \mathrm{mM}$ considering that the majority of phosphate ions are embedded in PNS. Thus, we determine that one $\mathrm{Ca}^{2+}$ ion interacts with $2.8(\sim 3) \mathrm{P}_{\mathrm{i}}$, forming PNS as 'calcium triphosphate units'.

Interestingly, we also observed a small $\mathrm{pH}$ variation strongly correlated with the free $\mathrm{Ca}^{2+}$ species (Fig. 6). The $\mathrm{pH}$ increases from 7.42 to $7.56(\Delta \mathrm{pH}=$ $0.14)$, reaching a plateau after $16 \mathrm{~h}$ similar to the concentration of free $\mathrm{Ca}^{2+}$.

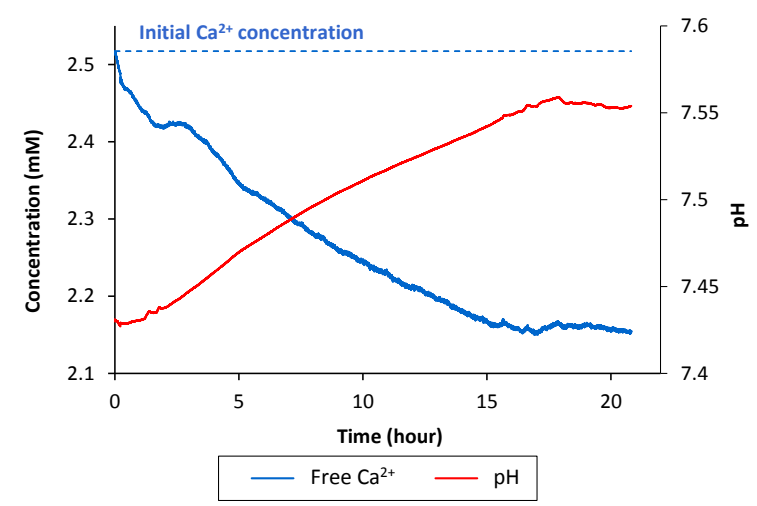

Figure 6: (A) Evolution of free $\mathrm{Ca}^{2+}$ concentration (blue curve) and $\mathrm{pH}$ (red curve) of mSBF over time. Concentration curve were temperature-corrected to mitigate biases in apparent concentrations.

This observation is important as the $\mathrm{pH}$ influences the phosphate speciation. The $\mathrm{pH}$ values suggest that PNS tend to accommodate both $\mathrm{HPO}_{4}{ }^{2-}$ and $\mathrm{H}_{2} \mathrm{PO}_{4}^{-}$ions but with a higher proportion of the latter $\left(\left[\mathrm{H}_{2} \mathrm{PO}_{4}{ }^{-}\right] /\left[\mathrm{HPO}_{4}{ }^{2-}\right]=0.36\right)$ (Fig. S5).

Considering relative contributions of bound $\mathrm{H}_{2} \mathrm{PO}_{4}{ }^{-}$ and $\mathrm{HPO}_{4}{ }^{2-}$ of $36 \%$ and $64 \%$ respectively, the PNS basic unit amounts to average nominal composition of $\left[\mathrm{Ca}\left(\mathrm{H}_{2} \mathrm{PO}_{4}\right)_{1.04}\left(\mathrm{HPO}_{4}\right)_{1.76}\right]_{n}{ }^{2.56-}$ (not considering coordinated water molecules), very similar to the calcium triphosphate units proposed by Habraken et al. ${ }^{27}$

\section{Conclusions}

Our data show that mSBF is a highly dynamic system that undergoes a continuous transformation after its preparation. It can be considered as stable or metastable, only after an extended equilibration period of $>24 \mathrm{~h}$. Prior, the dynamic emergence of calcium phosphate prenucleation species continuously changes the nanoscopic constitution of the solution.

Fig. 7 summarizes the picture we developed of PNS in $\mathrm{mSBF}$ from individual ions to small clusters to larger suprastructures. Initially, PNS solute species of $\sim 2 \mathrm{~nm}$ in diameter form right after $\mathrm{mSBF}$ preparation and experience accelerating phosphate exchange with maturation time. After equilibration, $\mathrm{mSBF}$ is found depleted of free phosphate as PNS form with an estimated $\mathrm{Ca} / \mathrm{P}$ molar ratio of $1 / 3$.

Given its widespread use, the understanding of SBF and PNS dispersed therein might be useful in a wide array of research fields. Among other examples, the investigation of non-classical crystallization pathways in the field of biomineralization or the development of novel bone-graft replacements rely heavily on SBF use.

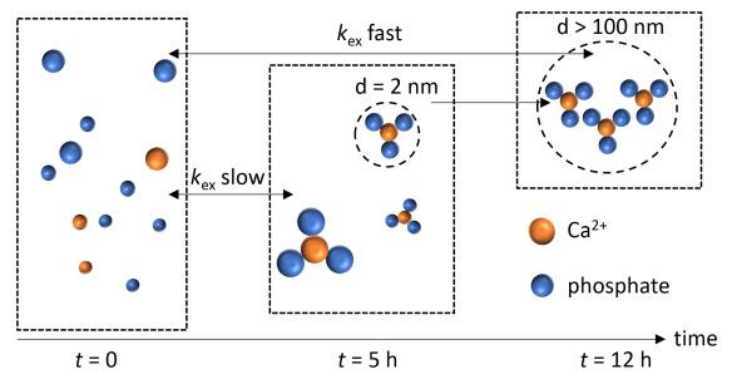

Figure 7. Scheme summarizing the PNS evolution in mSBF. After preparation, the ions are freely dispersed in solution. After $5 \mathrm{~h}$, they aggregate to from CaP species with a diameter of ca. 2-3 $\mathrm{nm}$. The exchange rate of phosphates between free and bound states is slow (on the NMR timescale) at this stage. After $12 \mathrm{~h}$, the $\mathrm{CaP}$ clusters can aggregate to form suprastructures of more than $100 \mathrm{~nm}$ in size where individual PNS are still present. The phosphate exchange is fast at this stage. The PNS are constituted by three phosphate units per $\mathrm{Ca}^{2+}$ ion.

The combination of NMR, Cryo-TEM, and $\mathrm{Ca}^{2+}$ titration thereby provides the possibility to trace the transformation of $P_{i}$ into $P_{P N S}$ in real-time and quantify the size and composition of PNS. Therefore, the presented integrative methodology might open new avenues for spatiotemporally resolved data sets the enable characterization of nanoscale inhomogeneiteis in solution and associated crystallization events at an improved level of detail.

\section{Experimental}

Sample preparation

Modified simulated body fluids (mSBF) were prepared following the protocol by Oyane et al. ${ }^{43,44}$ In brief, neat water was stirred at $37^{\circ} \mathrm{C}$, and the salts constituting $\mathrm{mSBF}$ were added successively to achieve concentrations comparable 
to those found in human blood plasma and in particular, a Pi concentration of $1 \mathrm{mM}$. After the addition of all the salts, the $\mathrm{pH}$ was adjusted to 7.4 with using $1.0 \mathrm{M} \mathrm{NaOH}$. After preparation, the mSBF was immediately transferred to an NMR tube, and $10 \% \mathrm{v} / \mathrm{v} \mathrm{D}_{2} \mathrm{O}$ were added as lock solvent.

\section{NMR Spectroscopy}

All NMR data were acquired at $37^{\circ} \mathrm{C}$ on a Bruker NEO NMR spectrometer operating at $11.7 \mathrm{~T}$ (500 $\mathrm{MHz}$ proton Larmor frequency) equipped with a 5 $\mathrm{mm}$ Bruker Prodigy BBO cryo-probe. For real-time detection, 1D ${ }^{31} \mathrm{P}$ NMR spectra were acquired every 7.5 minutes by averaging 64 FIDs. For detection $90^{\circ}$ flip-angle pulses were applied with a length of $12 \mu \mathrm{s}$ every $5 \mathrm{~s}$. Prior to Fourier transformation all data were zero filled and apodized using a Gaussian window function. Subsequent to Fourier transformation all data were baseline corrected. To extract signal intensities, the NMR signals were fitted to two Lorentzian functions using home written scripts embedded in the MATLAB software package using the 'fitNlorentzian' function.

For diffusion ordered spectroscopy (DOSY), spectra were acquired using the dstebpgp3s pulse sequence $^{49}$ embedded in the Bruker TopSpin 4 pulse sequence library. Spectra were acquired over $5 \mathrm{~h} 15$ by averaging 150 FIDs. For detection $90^{\circ}$ flipangle pulses were applied with a length of $12 \mu \mathrm{s}$ every 10 s. A 6 step z-gradient ramp was used with $2,14,25,37,48$ and $60 \%$ of the maximum gradient strength.

Data were analyzed using the GNAT software package $^{50}$ for MATLAB. Prior to Fourier transformation all data were zero filled and apodized using a Lorentzian window function. Subsequent to Fourier transformation all data were baseline corrected. Diffusion coefficients were extracted using mono-exponential fits to the Stejskal-Tanner equation. ${ }^{51}$

\section{Calcium ion potentiometry}

Calcium concentration and $\mathrm{pH}$ were monitored using a calcium-sensitive electrode (Ca-ISE, Metrohm) and a pH glass electrode (Unitrode, Metrohm) connected to a Titrando ${ }^{\mathrm{TM}}$ titration device (Metrohm) and analyzed with Tiamo ${ }^{\text {TM }}$ software (Metrohm). The Ca-ISE was calibrated at room temperature using 3 modified SBF-like solutions with respective calcium concentration of $0.1,1$ and $3 \mathrm{mM}$. These $\mathrm{m}$-SBF-like solutions were synthetized by following the procedure of Oyane et al. $^{43,44}$ omitting phosphates salts. For each solution, potential (in $\mathrm{mV}$ ) was measured and automatically fitted to the Nernst equation with a least square algorithm. The $\mathrm{pH}$-electrode was calibrated using 3 buffered solutions at $\mathrm{pH} 4, \mathrm{pH} 7$ and $\mathrm{pH} 9$ (Metrohm). Sample evolution was monitored for 24 hours recording potential every $10 \mathrm{~s}$ under gentle stirring.

\section{Cryo electron microscopy \& XEDS}

$\mathrm{m}$-SBF solutions were vitrified at specific time points after preparation (1, 5.5 and 23 hours). The morphology and the size of nanoparticles were determined from Cryo-Transmission Electron Microscopy (cryo-TEM) images. A drop $(3 \mu \mathrm{l})$ of solution was deposited on "quantifoil" (Quantifoil Micro Tools $\mathrm{GmbH}$, Germany) carbon membrane grids. The excess of liquid on the grid was absorbed with a filter paper and the grid was quench-frozen quickly in liquid ethane to form a thin vitreous ice film. The samples were transferred in the microscope and observed at low temperature ($\left.180{ }^{\circ} \mathrm{C}\right)$. Cryo-TEM images were recorded on ultrascan 1000, 2k x 2k CCD camera (Gatan, USA), using a LaB $_{6}$ JEOL JEM2100 (JEOL, Japan) cryo microscope operating at $200 \mathrm{kV}$ with a JEOL low dose system (Minimum Dose System, MDS) to protect the thin ice film from any irradiation before imaging and reduce the irradiation during the image capture.

X-ray Energy Dispersive Spectra (XEDS) characterizing the elemental composition of the samples have been recorded with a JEOL (Japan) XEDS detector with $140 \mathrm{eV}$ resolution using a JEOL (Japan) 2100F, Field Emission Gun instrument operating at $200 \mathrm{kV}$ under cryo condition. Images were recorded on UltraScan 4000 Gatan (USA) Camera with a $4096 \times 4096$ CCD.

\section{Supporting Information}

Reference NMR experiments, supplementary cryoTEM.

\section{Acknowledgements}

The authors acknowledge support by the NMR core facility of the Faculty of Chemistry, University Vienna. The authors thank Dr. Gregory L. Olsen for his support.

The project leading to this application received funding from the European Research Council (ERC) under the European Union's Horizon 2020 research and innovation programme (grant agreement 801936). This project was further supported by an FWF stand-alone grant (no. P-33338 N).

TA and TG thank CNRS-NTU "Excellence Science" Joint Research Program for funding.

\section{Notes}

The authors declare no competing financial interest. 


\section{REFERENCES}

(1) Lowenstam, H. A.; Lowenstam, P. E. D. of G. and P. S. H. a; Weiner, S.; Weiner, A. P. I. D. S. On Biomineralization; Oxford University Press, 1989.

(2) Glimcher, M. J. The Nature of the Mineral Phase in Bone: Biological and Clinical Implications. In Metabolic bone disease and clinically related disorders; Elsevier, 1998; pp 23-52e.

(3) Beniash, E. Biominerals-Hierarchical Nanocomposites: The Example of Bone. WIRES Nanomedicine Nanobiotechnology 2011, 3 (1), 47-69. https://doi.org/10.1002/wnan.105.

(4) Wang, W.; Yeung, K. W. K. Bone Grafts and Biomaterials Substitutes for Bone Defect Repair: A Review. Bioact. Mater. 2017, 2 (4), 224-247. https://doi.org/10.1016/j.bioactmat.2017.05.007.

(5) Mann, S. Biomineralization: Principles and Concepts in Bioinorganic Materials Chemistry; Oxford University Press, 2001.

(6) Meldrum, F. C.; Cölfen, H. Controlling Mineral Morphologies and Structures in Biological and Synthetic Systems. Chem. Rev. 2008, 108 (11), 4332-4432. https://doi.org/10.1021/cr8002856.

(7) Kokubo, T.; Kushitani, H.; Sakka, S.; Kitsugi, T.; Yamamuro, T. Solutions Able to Reproduce in Vivo Surface-Structure Changes in Bioactive Glass-Ceramic AW3. J. Biomed. Mater. Res. 1990, 24 (6), 721-734. https://doi.org/10.1002/jbm.820240607.

(8) Ohtsuki, C.; Kokubo, T.; Yamamuro, T. Mechanism of Apatite Formation on CaOSiO2P2O5 Glasses in a Simulated Body Fluid. J. Non-Cryst. Solids 1992, 143, 84-92.

(9) Zou, F.; Jiang, J.; Lv, F.; Xia, X.; Ma, X. Preparation of Antibacterial and Osteoconductive 3DPrinted PLGA/Cu(I)@ZIF-8 Nanocomposite Scaffolds for Infected Bone Repair. J. Nanobiotechnology 2020, 18 (1), 39. https://doi.org/10.1186/s12951-020-00594-6.

(10) Shin, K.; Jayasuriya, A. C.; Kohn, D. H. Effect of Ionic Activity Products on the Structure and Composition of Mineral Self Assembled on Three-Dimensional Poly(Lactide-Co-Glycolide) Scaffolds. J. Biomed. Mater. Res. A 2007, 83A (4), 1076-1086. https://doi.org/10.1002/jbm.a.31437.

(11) Misra, S. K.; Ansari, T. I.; Valappil, S. P.; Mohn, D.; Philip, S. E.; Stark, W. J.; Roy, I.; Knowles, J. C.; Salih, V.; Boccaccini, A. R. Poly(3-Hydroxybutyrate)
Multifunctional Composite Scaffolds for Tissue Engineering Applications. Biomaterials 2010, 31 (10), 2806-2815.

https://doi.org/10.1016/j.biomaterials.2009.12.045.

(12) Suárez-González, D.; Barnhart, K.; Saito, E.; Vanderby, R.; Hollister, S. J.; Murphy, W. L. Controlled Nucleation of Hydroxyapatite on Alginate Scaffolds for Stem Cell-Based Bone Tissue Engineering. J. Biomed. Mater. Res. A 2010, 95A (1), 222-234. https://doi.org/10.1002/jbm.a.32833.

(13) Yang, X.; Hutchinson, C. R. Corrosion-Wear of $\beta$-Ti Alloy TMZF (Ti-12Mo-6Zr-2Fe) in Simulated Body Fluid. Acta Biomater. 2016, 42, 429-439. https://doi.org/10.1016/j.actbio.2016.07.008.

(14) Jafari, S.; Singh Raman, R. K. In-Vitro Biodegradation and Corrosion-Assisted Cracking of a Coated Magnesium Alloy in Modified-Simulated Body Fluid. Mater. Sci. Eng. C 2017, 78, 278-287. https://doi.org/10.1016/j.msec.2017.04.079.

(15) Prabhu, D. B.; Nampoothiri, J.; Elakkiya, V.; Narmadha, R.; Selvakumar, R.; Sivasubramanian, R.; Gopalakrishnan, P.; Ravi, K. R. Elucidating the Role of Microstructural Modification on Stress Corrosion Cracking of Biodegradable Mg4Zn Alloy in Simulated Body Fluid. Mater. Sci. Eng. C 2020, 106, 110164. https://doi.org/10.1016/j.msec.2019.110164.

(16) Kaur, B.; Srivastava, R.; Satpati, B.; Kondepudi, K. K.; Bishnoi, M. Biomineralization of Hydroxyapatite in Silver lon-Exchanged Nanocrystalline ZSM-5 Zeolite Using Simulated Body Fluid. Colloids Surf. B Biointerfaces 2015, 135, 201-208.

https://doi.org/10.1016/j.colsurfb.2015.07.068.

(17) Elmi, M. M.; Elmi, F.; Sekineh Khazaee, P. Preparation and Characterization of Marine Mineralized ASC Nanocomposites through Biomineralization. Int. J. Biol. Macromol. 2019, 123, 817-825. https://doi.org/10.1016/j.ijbiomac.2018.11.131.

(18) Kokubo, T.; Takadama, H. How Useful Is SBF in Predicting in Vivo Bone Bioactivity? Biomaterials 2006, 27 (15), 2907-2915. https://doi.org/10.1016/j.biomaterials.2006.01.017.

(19) Bohner, M.; Lemaitre, J. Can Bioactivity Be Tested in Vitro with SBF Solution? Biomaterials 2009, 30 (12), 2175-2179. https://doi.org/10.1016/j.biomaterials.2009.01.008.

(20) Baino, F.; Yamaguchi, S. The Use of Simulated Body Fluid (SBF) for Assessing Materials Bioactivity in the Context of Tissue Engineering: Review and Challenges. 
Biomimetics 2020, 5 (4), 57.

https://doi.org/10.3390/biomimetics5040057.

(21) He, K.; Sawczyk, M.; Liu, C.; Yuan, Y.; Song, B.; Deivanayagam, R.; Nie, A.; Hu, X.; Dravid, V. P.; Lu, J.; Sukotjo, C.; Lu, Y.; Král, P.; Shokuhfar, T.; ShahbazianYassar, R. Revealing Nanoscale Mineralization Pathways of Hydroxyapatite Using in Situ Liquid Cell Transmission Electron Microscopy. Sci. Adv. 2020, 6 (47), eaaz7524. https://doi.org/10.1126/sciadv.aaz7524.

(22) Gebauer, D.; Wolf, S. E. Designing Solid Materials from Their Solute State: A Shift in Paradigms toward a Holistic Approach in Functional Materials Chemistry. J. Am. Chem. Soc. 2019, 141 (11), 4490-4504. https://doi.org/10.1021/jacs.8b13231.

(23) Dey, A.; Bomans, P. H. H.; Müller, F. A.; Will, J.; Frederik, P. M.; de With, G.; Sommerdijk, N. A. J. M. The Role of Prenucleation Clusters in Surface-Induced Calcium Phosphate Crystallization. Nat. Mater. 2010, 9 (12), 1010-1014. https://doi.org/10.1038/nmat2900.

(24) Nudelman, F.; Pieterse, K.; George, A.; Bomans, P. H. H.; Friedrich, H.; Brylka, L. J.; Hilbers, P. A. J.; de With, G.; Sommerdijk, N. A. J. M. The Role of Collagen in Bone Apatite Formation in the Presence of Hydroxyapatite Nucleation Inhibitors. Nat. Mater. 2010, 9 (12), 1004-1009. https://doi.org/10.1038/nmat2875.

(25) Gebauer, D.; Kellermeier, M.; D. Gale, J.; Bergström, L.; Cölfen, H. Pre-Nucleation Clusters as Solute Precursors in Crystallisation. Chem. Soc. Rev. 2014, 43 (7), 2348-2371. https://doi.org/10.1039/C3CS60451A.

(26) Carino, A.; Ludwig, C.; Cervellino, A.; Müller, E.; Testino, A. Formation and Transformation of Calcium Phosphate Phases under Biologically Relevant Conditions: Experiments and Modelling. Acta Biomater. 2018, 74, 478-488.

https://doi.org/10.1016/j.actbio.2018.05.027.

(27) Habraken, W. J. E. M.; Tao, J.; Brylka, L. J.; Friedrich, H.; Bertinetti, L.; Schenk, A. S.; Verch, A.; Dmitrovic, V.; Bomans, P. H. H.; Frederik, P. M.; Laven, J.; van der Schoot, P.; Aichmayer, B.; de With, G.; DeYoreo, J. J.; Sommerdijk, N. A. J. M. Ion-Association Complexes Unite Classical and Non-Classical Theories for the Biomimetic Nucleation of Calcium Phosphate. Nat. Commun. 2013, 4 (1), 1507. https://doi.org/10.1038/ncomms2490.

(28) Zhang, Q.; Jiang, Y.; Gou, B.-D.; Huang, J.; Gao, Y.-X.; Zhao, J.-T.; Zheng, L.; Zhao, Y.-D.; Zhang, T.-L.; Wang, K. In Situ Detection of Calcium Phosphate Clusters in Solution and Wet Amorphous Phase by Synchrotron X-
Ray Absorption Near-Edge Spectroscopy at Calcium KEdge. Cryst. Growth Des. 2015, 15 (5), 2204-2210. https://doi.org/10.1021/cg5018505.

(29) Wang, L.; Li, S.; Ruiz-Agudo, E.; Putnis, C. V.; Putnis, A. Posner's Cluster Revisited: Direct Imaging of Nucleation and Growth of Nanoscale Calcium Phosphate Clusters at the Calcite-Water Interface. CrystEngComm 2012, 14 (19), 6252-6256. https://doi.org/10.1039/C2CE25669J.

(30) Gebauer, D.; Völkel, A.; Cölfen, H. Stable Prenucleation Calcium Carbonate Clusters. Science 2008 322 (5909), 1819-1822.

https://doi.org/10.1126/science.1164271.

(31) Gebauer, D.; Cölfen, H. Prenucleation Clusters and Non-Classical Nucleation. Nano Today 2011, 6 (6), 564-584. https://doi.org/10.1016/j.nantod.2011.10.005.

(32) Pouget, E. M.; Bomans, P. H. H.; Goos, J. A. C. M.; Frederik, P. M.; With, G. de; Sommerdijk, N. A. J. M. The Initial Stages of Template-Controlled $\mathrm{CaCO} 3$ Formation Revealed by Cryo-TEM. Science 2009, 323 (5920), 1455-1458. https://doi.org/10.1126/science.1169434.

(33) Mohammed, A. S. A.; Carino, A.; Testino, A.; Andalibi, M. R.; Cervellino, A. In Situ Liquid SAXS Studies on the Early Stage of Calcium Carbonate Formation. Part. Part. Syst. Charact. 2019, 36 (6), 1800482. https://doi.org/10.1002/ppsc.201800482.

(34) Scheck, J.; Wu, B.; Drechsler, M.; Rosenberg R.; Van Driessche, A. E. S.; Stawski, T. M.; Gebauer, D. The Molecular Mechanism of Iron(III) Oxide Nucleation. J. Phys. Chem. Lett. 2016, 7 (16), 3123-3130. https://doi.org/10.1021/acs.jpclett.6b01237.

(35) E. Bonnett, P.; J. Carpenter, K.; Dawson, S.; J. Davey, R. Solution Crystallisation via a Submerged Liquid-Liquid Phase Boundary: Oiling Out. Chem. Commun. 2003, 0 (6), 698-699. https://doi.org/10.1039/B212062C.

(36) Peng, Y.; Wang, F.; Wang, Z.; Alsayed, A. M.; Zhang, Z.; Yodh, A. G.; Han, Y. Two-Step Nucleation Mechanism in Solid-Solid Phase Transitions. Nat. Mater. 2015, 14 (1), 101-108. https://doi.org/10.1038/nmat4083.

(37) Zahn, D. Thermodynamics and Kinetics of Prenucleation Clusters, Classical and Non-Classical Nucleation. ChemPhysChem 2015, 16 (10), 2069-2075. https://doi.org/10.1002/cphc.201500231. 
(38) Carino, A.; Testino, A.; Andalibi, M. R.; Pilger, F.; Bowen, P.; Ludwig, C. Thermodynamic-Kinetic Precipitation Modeling. A Case Study: The Amorphous Calcium Carbonate (ACC) Precipitation Pathway Unravelled. Cryst. Growth Des. 2017, 17 (4), 2006-2015. https://doi.org/10.1021/acs.cgd.7b00006.

(39) Jehannin, M.; Rao, A.; Cölfen, H. New Horizons of Nonclassical Crystallization. J. Am. Chem. Soc. 2019, 141 (26), 10120-10136.

https://doi.org/10.1021/jacs.9b01883.

(40) Wang, L.; Nancollas, G. H. Calcium Orthophosphates: Crystallization and Dissolution. Chem. Rev. 2008, 108 (11), 4628-4669. https://doi.org/10.1021/cr0782574.

(41) G. Vekilov, P. The Two-Step Mechanism of Nucleation of Crystals in Solution. Nanoscale 2010, 2 (11), 2346-2357. https://doi.org/10.1039/CONR00628A.

(42) Li, P.; Nakanishi, K.; Kokubo, T.; de Groot, K. Induction and Morphology of Hydroxyapatite, Precipitated from Metastable Simulated Body Fluids on Sol-Gel Prepared Silica. Biomaterials 1993, 14 (13), 963968. https://doi.org/10.1016/0142-9612(93)90186-6.

(43) Oyane, A.; Kim, H.-M.; Furuya, T.; Kokubo, T.; Miyazaki, T.; Nakamura, T. Preparation and Assessment of Revised Simulated Body Fluids. J. Biomed. Mater. Res. A 2003, 65A (2), 188-195.

https://doi.org/10.1002/jbm.a.10482.

(44) Oyane, A.; Onuma, K.; Ito, A.; Kim, H.-M.; Kokubo, T.; Nakamura, T. Formation and Growth of Clusters in Conventional and New Kinds of Simulated Body Fluids. J. Biomed. Mater. Res. A 2003, 64A (2), 339348. https://doi.org/10.1002/jbm.a.10426.

(45) Ernst, R. R.; Bodenhausen, G.; Wokaun, A. Principles of Nuclear Magnetic Resonance in One and Two Dimensions; Clarendon press Oxford, 1987; Vol. 14.

(46) Norris, J. R. Rapid Computation of Magnetic Resonance Line Shapes for Exchange among Many Sites. Chem. Phys. Lett. 1967, 1 (8), 333-334. https://doi.org/10.1016/0009-2614(67)80009-5.

(47) Carver, J. P.; Richards, R. E. A General Two-Site Solution for the Chemical Exchange Produced Dependence of T2 upon the Carr-Purcell Pulse Separation. J. Magn. Reson. 1969 1972, 6 (1), 89-105. https://doi.org/10.1016/0022-2364(72)90090-X.

(48) Weber, E. M. M.; Kress, T.; Abergel, D.; Sewsurn, S.; Azaïs, T.; Kurzbach, D. Assessing the Onset of Calcium Phosphate Nucleation by Hyperpolarized
Real-Time NMR. Anal. Chem. 2020, 92 (11), 7666-7673. https://doi.org/10.1021/acs.analchem.0c00516.

(49) Jerschow, A. Thermal Convection Currents in NMR: Flow Profiles and Implications for Coherence Pathway Selection. J. Magn. Reson. 2000, 145 (1), 125131. https://doi.org/10.1006/jmre.2000.2083.

(50) Castañar, L.; Poggetto, G. D.; Colbourne, A. A.; Morris, G. A.; Nilsson, M. The GNAT: A New Tool for Processing NMR Data. Magn. Reson. Chem. 2018, 56 (6), 546-558. https://doi.org/10.1002/mrc.4717.

(51) Borkowski, K.; Krzyżak, A. T. The Generalized Stejskal-Tanner Equation for Non-Uniform Magnetic Field Gradients. J. Magn. Reson. 2018, 296, 23-28. https://doi.org/10.1016/j.jmr.2018.08.010. 